\title{
Kirszbraun's Theorem via an Explicit Formula
}

\author{
Daniel Azagra, Erwan Le Gruyer, and Carlos Mudarra
}

Abstract. Let $X, Y$ be two Hilbert spaces, let $E$ be a subset of $X$, and let $G: E \rightarrow Y$ be a Lipschitz mapping. A famous theorem of Kirszbraun's states that there exists $\tilde{G}: X \rightarrow Y$ with $\tilde{G}=G$ on $E$ and $\operatorname{Lip}(\tilde{G})=\operatorname{Lip}(G)$. In this note we show that in fact the function $\tilde{G}:=\nabla_{Y}(\operatorname{conv}(g))(\cdot, 0)$, where

$$
g(x, y)=\inf _{z \in E}\left\{\langle G(z), y\rangle+\frac{\operatorname{Lip}(G)}{2}\|(x-z, y)\|^{2}\right\}+\frac{\operatorname{Lip}(G)}{2}\|(x, y)\|^{2},
$$

defines such an extension. We apply this formula to get an extension result for strongly biLipschitz mappings. Related to the latter, we also consider extensions of $C^{1,1}$ strongly convex functions.

\section{An Explicit Formula for Kirszbraun's Theorem}

In 1934, M. D. Kirszbraun [23] proved that, for every subset $E$ of $\mathbb{R}^{n}$ and every Lipschitz function $f: E \rightarrow \mathbb{R}^{m}$, there exists a Lipschitz extension $F: \mathbb{R}^{n} \rightarrow \mathbb{R}^{m}$ of $f$ such that $\operatorname{Lip}(F)=\operatorname{Lip}(f)$. Here, $\operatorname{Lip}(\varphi)$ denotes the Lipschitz constant of $\varphi$, that is,

$$
\operatorname{Lip}(\varphi)=\sup _{x \neq y} \frac{\|\varphi(x)-\varphi(y)\|}{\|x-y\|} .
$$

This theorem was generalized for Hilbert spaces $X, Y$ in place of $\mathbb{R}^{n}$ and $\mathbb{R}^{m}$ by F. A. Valentine [30] in 1945, and the result is often referred to as the KirszbraunValentine theorem. The proof is rather nonconstructive, in the sense that it requires the use of Zorn's lemma or transfinite induction at least in the nonseparable case. In the separable case, the proof can be made by induction, considering a dense sequence $\left\{x_{k}\right\}$ in $X$ and at each step managing to extend $f$ from $E \cup\left\{x_{1}, \ldots, x_{m}\right\}$ to $E \cup\left\{x_{1}, \ldots, x_{m+1}\right\}$ while preserving the Lipschitz constant of the extension by using Helly's theorem or intersection properties of families of balls, but it is still not clear what the extension looks like. Several other proofs and generalizations that are not

Received by the editors February 1, 2019; revised April 20, 2020.

Published online on Cambridge Core April 29, 2020.

D. Azagra and C. Mudarra were partially supported by Grant MTM2015-65825-P and by the Severo Ochoa Program for Centres of Excellence in R \& D (Grant SEV-2015-0554).

AMS subject classification: 47H09, 52A41, 54C20.

Keywords: Lipschitz function, Kirszbraun theorem. 
constructive either have appeared in the literature; see [26, 21, 15, 11, 27, 5, 1]; apart from Zorn's lemma or induction these proofs are based on intersection properties of arbitrary families of balls, or on maximal extensions of non-expansive operators and Fitzpatrick functions. In 2008, H. H. Bauschke and X. Wang [9] gave the first constructive proof of the Kirszbraun-Valentine theorem of which we are aware; they relied on their previous work [8] on extension and representation of monotone operators and the Fitzpatrick function. See also [2], where some of these techniques are used to construct definable versions of Helly's and Kirszbraun's theorems in arbitrary definably complete expansions of ordered fields. Finally, in 2015 E. Le Gruyer and T-V. Phan provided sup-inf explicit extension formulas for Lipschitz mappings between finite dimensional spaces by relying on Le Gruyer's solution to the minimal $C^{1,1}$ extension problem for 1-jets; see [25, Theorem 32 and 33] and [24].

In this note, we present a short proof of the Kirszbraun-Valentine theorem in which the extension is given by an explicit formula. This proof is based on our previous work concerning $C^{1,1}$ extensions of 1-jets with optimal Lipschitz constants of the gradients [4]. See [14] for an alternative construction of such $C^{1,1}$ extensions on the Hilbert space, and $[12,16,17,18]$ for the much more difficult question of extending functions (as opposed to jets) to $C^{1,1}$ or $C^{m, 1}$ functions on $\mathbb{R}^{n}$.

If $X$ is a Hilbert space, $E \subset X$ is an arbitrary subset, and $(f, G): E \rightarrow \mathbb{R} \times X$ is a 1jet on $E$, we will say that $(f, G)$ satisfies condition $\left(W^{1,1}\right)$ with constant $M>0$ on $E$ provided that

$$
f(y) \leq f(x)+\frac{1}{2}\langle G(x)+G(y), y-x\rangle+\frac{M}{4}\|x-y\|^{2}-\frac{1}{4 M}\|G(x)-G(y)\|^{2},
$$

for all $x, y \in E$.

In $[32,24]$, it was proved that condition $\left(W^{1,1}\right)$ with constant $M>0$ is a necessary and sufficient condition on $f: E \rightarrow \mathbb{R}, G: E \rightarrow X$ for the existence of a function $F \in$ $C^{1,1}(X)$ with $\operatorname{Lip}(\nabla F) \leq M$ and such that $F=f$ and $\nabla F=G$ on $E$. Here, $\nabla F(x)$ denotes the gradient of $F$ at the point $x$, that is, the unique vector $\nabla F(x) \in X$ for which $D F(x)(v)=\langle\nabla F(x), v\rangle$ for every $v \in X$, where $D F(x) \in X^{*}$ denotes the Fréchet derivative of $F$ at the point $x$. More recently, as a consequence of a similar extension theorem for $C^{1,1}$ convex functions, we have found an explicit formula for such an extension $F$.

Theorem 1.1 [4, Theorem 3.4] Let E be a subset of a Hilbert space X. Given a 1-jet $(f, G)$ satisfying condition $\left(W^{1,1}\right)$ with constant $M$ on $E$, the formula

$$
\begin{aligned}
F & =\operatorname{conv}(g)-\frac{M}{2}\|\cdot\|^{2}, \\
g(x) & =\inf _{y \in E}\left\{f(y)+\langle G(y), x-y\rangle+\frac{M}{2}\|x-y\|^{2}\right\}+\frac{M}{2}\|x\|^{2}, \quad x \in X,
\end{aligned}
$$

defines a $C^{1,1}(X)$ function with $F_{\left.\right|_{E}}=f,(\nabla F)_{\left.\right|_{E}}=G$, and $\operatorname{Lip}(\nabla F) \leq M$.

Here, $\operatorname{conv}(g)$ denotes the convex envelope of $g$, defined by

$$
\begin{aligned}
& \operatorname{conv}(g)(x)= \\
& \sup \{h(x): h \text { is convex, proper and lower semicontinuous, } h \leq g\} .
\end{aligned}
$$


Another expression for $\operatorname{conv}(g)$ is given by

$$
\begin{aligned}
& \operatorname{conv}(g)(x)= \\
& \quad \inf \left\{\sum_{j=1}^{k} \lambda_{j} g\left(x_{j}\right): \lambda_{j} \geq 0, \sum_{j=1}^{k} \lambda_{j}=1, x=\sum_{j=1}^{k} \lambda_{j} x_{j}, k \in \mathbb{N}\right\},
\end{aligned}
$$

and also by the Fenchel biconjugate of $g$, that is,

$$
\operatorname{conv}(g)=g^{* *}
$$

where

$$
h^{*}(x):=\sup _{v \in X}\{\langle v, x\rangle-h(v)\} ;
$$

see, for instance, [13, Proposition 4.4.3]. In the case where $X$ is finite dimensional, say $X=\mathbb{R}^{n}$, the expression (1.3) can be made simpler: by using Carathéodory's Theorem one can show that it is enough to consider convex combinations of at most $n+1$ points. That is to say, if $g: \mathbb{R}^{n} \rightarrow \mathbb{R}$, then

$$
\operatorname{conv}(g)(x)=\inf \left\{\sum_{j=1}^{n+1} \lambda_{j} g\left(x_{j}\right): \lambda_{j} \geq 0, \sum_{j=1}^{n+1} \lambda_{j}=1, x=\sum_{j=1}^{n+1} \lambda_{j} x_{j}\right\} ;
$$

see, for instance, [28, Corollary 17.1.5].

In general, the convex envelope does not preserve smoothness of orders higher than $C^{1}$ and $C^{1,1}$. For instance, the function $g(x, y)=\sqrt{x^{2}+e^{-y^{2}}}$ defined on $\mathbb{R}^{2}$ is real analytic and its convex envelope is $\operatorname{conv}(g)(x, y)=|x|$ for every $(x, y) \in \mathbb{R}^{2}$; see [10]. In [22], Kirchheim and Kristensen proved that the convex envelope of a differentiable function $g$ on $\mathbb{R}^{n}$ is of class $C^{1}$, provided that $g$ is coercive. On the other hand, if $g$ is of class $C^{1,1}$ on a Hilbert space $X$, or even if $g$ only satisfies

$$
g(x+h)+g(x-h)-2 g(x) \leq M\|h\|^{2}, \quad x, h \in X
$$

for some $M>0$, then $\operatorname{conv}(g)$ is of class $C^{1,1}$ and $\operatorname{Lip}(\nabla \operatorname{conv}(g)) \leq M$; see [4, Theorem 2.3]. In particular, if $g=\inf _{i}\left(g_{i}\right)$ is the infimum of an arbitrary family of parabolas $g_{i}$, whose second derivatives are uniformly bounded by a constant $M>0$, then $\operatorname{conv}(g)$ is of class $C^{1,1}$ with $\operatorname{Lip}(\nabla \operatorname{conv}(g)) \leq M$, provided that $g$ has a convex lower semicontinuous minorant. However, $\operatorname{conv}(g)$ is not necessarily of class $C^{2}$ even when $g$ is the minimum of two parabolas: if we define $g(x)=\min \left\{x^{2},(x-1)^{2}\right\}$ for $x \in \mathbb{R}$, then $\operatorname{conv}(g)(x)=x^{2}$ for $x \leq 0, \operatorname{conv}(g)=0$ for $0 \leq x \leq 1$, and $\operatorname{conv}(g)=$ $(x-1)^{2}$ for $x \geq 1$; and therefore $\operatorname{conv}(g) \in C^{1,1}(\mathbb{R}) \backslash C^{2}(\mathbb{R})$.

Theorem 1.2 (Kirszbraun's theorem via an explicit formula.) Let $X, Y$ be two Hilbert spaces, let $E$ be a subset of $X$, and let $G: E \rightarrow Y$ be a Lipschitz mapping. There exists $\tilde{G}: X \rightarrow Y$ with $\tilde{G}=G$ on $E$ and $\operatorname{Lip}(\tilde{G})=\operatorname{Lip}(G)$. In fact, if $M=\operatorname{Lip}(G)$, then the function $\tilde{G}(x):=\nabla_{Y}(\operatorname{conv}(g))(x, 0), x \in X$, where

$$
g(x, y)=\inf _{z \in E}\left\{\langle G(z), y\rangle_{Y}+\frac{M}{2}\|x-z\|_{X}^{2}\right\}+\frac{M}{2}\|x\|_{X}^{2}+M\|y\|_{Y}^{2}, \quad(x, y) \in X \times Y,
$$

defines such an extension. 
Here, $\|\cdot\|_{X}$ and $\|\cdot\|_{Y}$ denote the norm on $X$ and $Y$, respectively. Also, the inner products in $X$ and $Y$ are denoted by $\langle\cdot, \cdot\rangle_{X}$ and $\langle\cdot, \cdot\rangle_{Y}$, respectively. For any function $F, \nabla_{Y} F$ will stand for the $Y$-partial derivatives of $F$, that is, the canonical projection from $X \times Y$ onto $Y$ composed with $\nabla F$.

Proof We consider on $X \times Y$ the norm given by $\|(x, y)\|=\sqrt{\|x\|_{X}^{2}+\|y\|_{Y}^{2}}$ for every $(x, y) \in X \times Y$. Then $X \times Y$ is a Hilbert space whose inner product is $\left\langle(x, y),\left(x^{\prime}, y^{\prime}\right)\right\rangle=\left\langle x, x^{\prime}\right\rangle_{X}+\left\langle y, y^{\prime}\right\rangle_{Y}$ for every $(x, y),\left(x^{\prime}, y^{\prime}\right) \in X \times Y$. We define the 1-jet $\left(f^{*}, G^{*}\right)$ on $E \times\{0\} \subset X \times Y$ by $f^{*}(x, 0)=0$ and $G^{*}(x, 0)=(0, G(x))$. Then the jet $\left(f^{*}, G^{*}\right)$ satisfies condition $\left(W^{1,1}\right)$ on $E \times\{0\}$ with constant $M$ (see inequality (1.1)). Indeed, by the definition of $f^{*}$, and $G^{*}$, we can write, for every $(x, 0),(y, 0) \in E \times\{0\}$,

$$
\begin{aligned}
f^{*}(x, 0) & -f^{*}(y, 0)+\frac{1}{2}\left\langle G^{*}(x, 0)+G^{*}(y, 0),(y, 0)-(x, 0)\right\rangle \\
& +\frac{M}{4}\|(x, 0)-(y, 0)\|^{2}-\frac{1}{4 M}\left\|G^{*}(x, 0)-G^{*}(y, 0)\right\|^{2} \\
= & \frac{1}{2}\langle(0, G(x))+(0, G(y)),(y, 0)-(x, 0)\rangle \\
& +\frac{M}{4}\|x-y\|_{X}^{2}-\frac{1}{4 M}\|G(x)-G(y)\|_{Y}^{2} \\
= & \frac{M}{4}\|x-y\|_{X}^{2}-\frac{1}{4 M}\|G(x)-G(y)\|_{Y}^{2},
\end{aligned}
$$

and the last term is nonnegative, because $G$ is $M$-Lipschitz on $E$.

Therefore, Theorem 1.1 asserts that the function $F$ defined by $F=\operatorname{conv}(g)-\frac{M}{2} \|$. $\|^{2}$, where

$$
\begin{array}{r}
g(x, y)=\inf _{z \in E}\left\{f^{*}(z, 0)+\left\langle G^{*}(z, 0),(x-z, y)\right\rangle+\frac{M}{2}\|(x-z, y)\|^{2}\right\} \\
+\frac{M}{2}\|(x, y)\|^{2},
\end{array}
$$

is of class $C^{1,1}(X \times Y)$ with $(F, \nabla F)=\left(f^{*}, G^{*}\right)$ on $E \times\{0\}$ and $\operatorname{Lip}(\nabla F) \leq M$. In particular, the mapping $X \ni x \mapsto \tilde{G}(x):=\nabla_{Y} F(x, 0) \in Y$ is $M$-Lipschitz and extends $G$ from $E$ to $X$. Finally, the expressions defining $\tilde{G}$ and $g$ can be simplified as

$$
\begin{aligned}
\tilde{G}(x) & =\nabla_{Y}\left(\operatorname{conv}(g)-\frac{M}{2}\|\cdot\|^{2}\right)(x, 0) \\
& =\nabla_{Y}(\operatorname{conv}(g))(x, 0)-\nabla_{Y}\left(\frac{M}{2}\|\cdot\|^{2}\right)(x, 0)=\nabla_{Y}(\operatorname{conv}(g))(x, 0)
\end{aligned}
$$

and

$$
g(x, y)=\inf _{z \in E}\left\{\langle G(z), y\rangle_{Y}+\frac{M}{2}\|x-z\|_{X}^{2}\right\}+\frac{M}{2}\|x\|_{X}^{2}+M\|y\|_{Y}^{2} .
$$

Let $X$ be a Hilbert space with inner product and associated norm denoted by $\langle\cdot, \cdot\rangle$ and $\|\cdot\|$, respectively. If $E \subset X$ is arbitrary and $G: E \rightarrow X$ is a mapping, we say that $G$ is firmly non-expansive if

$$
\langle G(x)-G(y), x-y\rangle \geq\|G(x)-G(y)\|^{2} \quad \text { for all } \quad x, y \in E .
$$

Important examples of firmly non-expansive mappings are the metric projections onto closed convex sets of Hilbert spaces and the proximal mappings $\operatorname{prox}_{f}: X \rightarrow X$ of proper lower semicontinuous convex functions $f: X \rightarrow(-\infty,+\infty]$; see [6, Chapter 12]. Firmly non-expansive mappings arise naturally in convex feasibility problems 
too: given a family $C_{1}, \ldots, C_{N}$ of closed convex sets of a Hilbert space, find a point $x \in \bigcap_{i} C_{i}$. Also, these mappings are known to be resolvents $J_{A}=(A+I)^{-1}$ of monotone or maximally monotone operators $A: X \rightrightarrows X$, and they play a crucial role in the following basic problem that arises in several branches of applied mathematics: given a maximally monotone operator $A$ : $X \rightrightarrows X$, find a point $x \in X$ with $0 \in A x$. For more information about firmly non-expansive mappings and their applications, see $[5,6,7,9]$ and the references therein.

It is well known that a mapping $G: E \rightarrow X$ is firmly non-expansive if and only $2 G-$ $I: E \rightarrow X$ is 1-Lipschitz, where $I$ denotes the identity map; see [6, Proposition 4.2] for a proof of this fact. Using this characterization and Theorem 1.2, we obtain the following corollary.

Corollary 1.3 Let $G: E \rightarrow X$ be a firmly non-expansive mapping defined on a subset $E$ of a Hilbert space $X$. Then $G$ can be extended to a firmly non-expansive mapping $\tilde{G}: X \rightarrow$ $X$ by means of the formula $\tilde{G}(x):=\frac{1}{2}\left(P_{2}(\nabla(\operatorname{conv}(g))(x, 0))+x\right), x \in X$, where

$$
\begin{aligned}
& P_{2}(x, y)=y, \quad(x, y) \in X \times X, \quad \text { and } \\
& g(x, y)=\inf _{z \in E}\left\{2\langle G(z), y\rangle+\frac{1}{2}\|z-(x+y)\|^{2}\right\}+\frac{1}{2}\|x-y\|^{2}, \quad(x, y) \in X \times X
\end{aligned}
$$

\section{Extensions of Strongly BiLipschitz Mappings}

In this section, we consider strongly biLipschitz mappings, which appear naturally as derivatives of strongly convex $C^{1,1}$ functions, and we provide an extension result for this class of mappings.

Definition 2.1. Let $E$ be a subset of a Hilbert space $X$. We say that a mapping $G: E \rightarrow$ $X$ is strongly biLipschitz provided that

$$
\operatorname{SBilip}(G):=\inf _{x, y \in E ; x \neq y} \frac{2\langle x-y, G(x)-G(y)\rangle}{\|x-y\|^{2}+\|G(x)-G(y)\|^{2}}>0 .
$$

Strongly biLipschitz mappings may be interesting with regard to some problems in computer vision or image processing where one needs to match points in $\mathbb{R}^{n}$ : for instance, given two sets of points in $\mathbb{R}^{n}$ with equal cardinality, find a homeomorphism from $\mathbb{R}^{n}$ onto itself that does not distort distances too much and takes one set onto the other. Supposing that the data satisfy the strongly biLipschitz condition, our explicit formula for such an extension can be useful. Also, in [7, Corollary 4.5], it was shown that strongly biLipschitz mappings are closely related to contractive mappings: a maximally monotone mapping $G$ is strongly biLipschitz if and only if its reflected resolvent $N=2(G+I)^{-1}-I$ is a contractive mapping. See [29, Chapter 12] for more information about resolvent mappings and maximally monotone operators.

It should also be noted that it is not generally true that a biLipschitz mapping whose domain and range is a subset of the same Hilbert space $X$ extends to a total one-to-one continuous mapping, as shown by the following example. 
Example 2.2. Let $|\cdot|$ denote the euclidean norm on $\mathbb{R}^{n}$. Let $C=\left\{x \in \mathbb{R}^{n}:|x|=1\right\} \cup$ $\{p\}$, let $p$ be any point with $|p|>1$, and let $g: C \rightarrow \mathbb{R}^{n}$ be defined by $g(x)=x$ for $|x|=1$ and $g(p)=0$. Then both $g$ and $g^{-1}$ are Lipschitz but no continuous extension of $g$ to $\mathbb{R}^{n}$ can be one-to-one.

However, this is true for the class of strongly biLipschitz mappings, and moreover, the extension can be performed without increasing what seems natural to call the strong biLipschitz constant $\operatorname{SBilip}(G)$, as we will show by using the extension formula for Lipschitz mappings given by Theorem 1.2.

Proposition 2.3 If $G: E \rightarrow X$ is strongly biLipschitz, then $G$ is biLipschitz.

Proof For every $x, y \in E$, we have

$$
\begin{aligned}
2\|x-y\|\|G(x)-G(y)\| & \geq 2\langle x-y, G(x)-G(y)\rangle \\
& \geq \alpha\left(\|x-y\|^{2}+\|G(x)-G(y)\|^{2}\right),
\end{aligned}
$$

where $\alpha=\operatorname{SBilip}(G)$ is as in Definition 2.1. It follows that $G$ is one-to-one. Note that we can write (2.1) in the equivalent form

$$
\left\|G(x)-G(y)-\frac{1}{\alpha}(x-y)\right\|^{2} \leq \frac{1-\alpha^{2}}{\alpha^{2}}\|x-y\|^{2} .
$$

Setting $\lambda:=\frac{\|G(x)-G(y)\|}{\|x-y\|}$ for $x \neq y,(2.1)$ holds if and only if

$$
\lambda^{2}-\frac{2}{\alpha} \lambda+1 \leq 0
$$

which is equivalent to $\frac{1}{K} \leq \lambda \leq K$; where $K=\frac{1}{\alpha}+\left(\frac{1}{\alpha^{2}}-1\right)^{1 / 2}$. This means that for any $x, y \in E$ with $x \neq y$, we have

$$
\frac{1}{K} \leq \frac{\|G(x)-G(y)\|}{\|x-y\|} \leq K .
$$

Therefore, $G$ is a biLipschitz mapping.

Remark 2.4. (i) If $X=\mathbb{R}$, then the strongly biLipschitz functions are exactly the strictly increasing biLipschitz functions.

(ii) If $G: E \rightarrow X$ is such that $\operatorname{SBilip}(G)=1$, then $G$ is the restriction of a translation.

(iii) If $G: E \rightarrow X$ is an isometry such that

$$
\alpha:=\inf _{x, y \in E ; x \neq y} \frac{\langle x-y, G(x)-G(y)\rangle}{\|x-y\|^{2}}>0,
$$

then $G$ is a strongly biLipschitz function with $\operatorname{SBilip}(G)=\alpha$. However, the composition of strongly biLipschitz isometries need not be strongly biLipschitz (for instance, if $r: \mathbb{R}^{2} \rightarrow \mathbb{R}^{2}$ is defined by $r(z)=e^{\pi i / 4} z$, then $r$ is strongly biLipschitz, but $r^{2}$ is not (and in fact $r^{4}=-$ id is not strongly biLipschitz locally on any disk). 
Theorem 2.5 Let $G: E \rightarrow X$ be a strongly biLipschitz mapping. Then $G$ extends to a strongly biLipschitz mapping on $X$ preserving the strongly bilipchitz constant $\operatorname{SBilip}(G)$. Moreover, if $\alpha=\operatorname{SBilip}(G)$, the formula $\tilde{G}(x):=P_{2}(\nabla(\operatorname{conv}(g))(x, 0))+\frac{1}{\alpha} x, x \in X$; where

$$
\begin{aligned}
P_{2}(x, y)= & y, \quad(x, y) \in X \times X, \quad \text { and } \\
g(x, y)= & \inf _{z \in E}\left\{\langle G(z), y\rangle-\frac{1}{\alpha}\langle z, y\rangle+\frac{\sqrt{1-\alpha^{2}}}{2 \alpha}\|x-z\|^{2}\right\} \\
& +\frac{\sqrt{1-\alpha^{2}}}{\alpha}\left(\frac{1}{2}\|x\|^{2}+\|y\|^{2}\right), \quad(x, y) \in X \times X,
\end{aligned}
$$

defines such an extension.

Proof We know from the characterization (2.2) that $G-\frac{1}{\alpha} I$ is Lipschitz on $E$ with $\operatorname{Lip}\left(G-\frac{1}{\alpha} I\right) \leq \sqrt{\frac{1-\alpha^{2}}{\alpha^{2}}}$. By Theorem 1.2, the mapping $T: X \rightarrow X$ defined as $T(x):=$ $P_{2}(\nabla(\operatorname{conv}(g))(x, 0)) x \in X$; where $P_{2}(x, y)=y$ for every $(x, y) \in X \times X$, and

$$
\begin{aligned}
g(x, y)= & \inf _{z \in E}\left\{\langle G(z), y\rangle-\frac{1}{\alpha}\langle z, y\rangle+\frac{\sqrt{1-\alpha^{2}}}{2 \alpha}\|x-z\|^{2}\right\} \\
& +\frac{\sqrt{1-\alpha^{2}}}{\alpha}\left(\frac{1}{2}\|x\|^{2}+\|y\|^{2}\right), \quad(x, y) \in X \times X,
\end{aligned}
$$

is an extension of $G-\frac{1}{\alpha} I$ to all of $X$ such that $\operatorname{Lip}(T)=\operatorname{Lip}\left(G-\frac{1}{\alpha} I\right) \leq \sqrt{\frac{1-\alpha^{2}}{\alpha^{2}}}$. Therefore, if we define the function $\tilde{G}=T+\frac{1}{\alpha} I$, we have that

$$
\left\|\tilde{G}(x)-\tilde{G}(y)-\frac{1}{\alpha}(x-y)\right\|^{2}=\|T(x)-T(y)\|^{2} \leq \frac{1-\alpha^{2}}{\alpha^{2}}\|x-y\|^{2}
$$

for all $x, y \in X$. We obtain from (2.2) that $\tilde{G}$ is strongly biLipschitz on $X$ with $\operatorname{SBilip}(\tilde{G})=\alpha$. Also, since $T$ is an extension of $G-\frac{1}{\alpha} I$, it is obvious that $\tilde{G}$ is an extension of $G$.

\section{$3 C^{1,1}$ Strongly Convex Functions}

In this section, we characterize the 1-jets that can be interpolated by strongly convex functions of class $C^{1,1}$ in Hilbert spaces. A function $F: X \rightarrow \mathbb{R}$ is strongly convex if $F-c\|\cdot\|^{2}$ is convex for some $c>0$. In Proposition 3.2, we will see that the gradient of a $C^{1,1}$ strongly convex function is a biLipschitz mapping. These functions arise naturally when studying smooth manifolds of positive curvature as well as in problems involving Monge-Ampère equations. See the papers [19, 20, 31] for some results and problems involving smooth strongly convex functions.

Throughout this section $X$ denotes a Hilbert space with norm and inner product denoted by $\langle\cdot, \cdot\rangle$ and $\|\cdot\|$, respectively.

Definition 3.1. Let $E \subseteq X$ be arbitrary, let $(f, G): E \rightarrow \mathbb{R} \times X$ be a 1-jet and $c \in$ $\mathbb{R}, M>0$ constants such that $M>c$. We say that $(f, G)$ satisfies condition $\left(S C W^{1,1}\right)$ with constants $(c, M)$ provided that 


$$
\begin{array}{r}
f(x) \geq f(y)+\langle G(y), x-y\rangle+\frac{c}{M-c}\langle G(x)-G(y), y-x\rangle+\frac{c M}{2(M-c)}\|x-y\|^{2} \\
+\frac{1}{2(M-c)}\|G(x)-G(y)\|^{2}
\end{array}
$$

for every $x, y \in E$.

Proposition 3.2 Assume that $(f, G): E \rightarrow \mathbb{R} \times X$ satisfies condition $\left(S C W^{1,1}\right)$ with constants $(c, M)$. Then the following properties hold.

(i) For every $x, y \in E$, we have

$$
(c+M)\langle G(x)-G(y), x-y\rangle \geq c M\|x-y\|^{2}+\|G(x)-G(y)\|^{2} .
$$

(ii) $G$ is Lipschitz with $c \leq \operatorname{Lip}(G) \leq M$.

(iii) If $c>0$, then $G$ is strongly biLipschitz with $\operatorname{SBilip}(G) \geq \frac{2}{c+M} \min \{1, c M\}$.

(iv) For $c=-M$ we recover Wells's condition $W^{1,1}$ considered in $[32,24,4]$. For $c=0$, $\left(S C W^{1,1}\right)$ is just condition $\left(C W^{1,1}\right)$ of $[3,4]$. For $c \in(0, M]$ we have what can be called a $C^{1,1}$ strongly convex 1-jet, which in the extreme case $c=M$ becomes the restriction of a quadratic function to $E$.

Proof (i) Let $x, y \in E$. By summing the inequalities

$$
\begin{aligned}
f(x) \geq & f(y)+\langle G(y), x-y\rangle+\frac{c}{M-c}\langle G(x)-G(y), y-x\rangle+\frac{c M}{2(M-c)}\|x-y\|^{2} \\
& +\frac{1}{2(M-c)}\|G(x)-G(y)\|^{2}, \\
f(y) \geq & f(x)+\langle G(x), y-x\rangle+\frac{c}{M-c}\langle G(y)-G(x), x-y\rangle+\frac{c M}{2(M-c)}\|x-y\|^{2} \\
& +\frac{1}{2(M-c)}\|G(x)-G(y)\|^{2},
\end{aligned}
$$

we obtain

$$
0 \geq\left(1+\frac{2 c}{M-c}\right)\langle G(x)-G(y), y-x\rangle+\frac{c M}{M-c}\|x-y\|^{2}+\frac{1}{M-c}\|G(x)-G(y)\|^{2},
$$

which is equivalent to the desired estimation.

(ii) Let $x, y \in E$ be such that $x \neq y$. Writing $\lambda=\|G(x)-G(y)\| /\|x-y\|$, the inequality in $(i)$ yields $\lambda^{2}-(c+M) \lambda+c M \leq 0$, which in turn implies $c \leq \lambda \leq M$.

(iii) It follows immediately from (i) and Definition 2.1.

We say that a 1-jet $(f, G): E \rightarrow \mathbb{R} \times X$ satisfies condition $\left(C W^{1,1}\right)$ with constant $M>0$ on $E$ provided that

$$
f(x) \geq f(y)+\langle G(y), x-y\rangle+\frac{1}{2 M}\|G(x)-G(y)\|^{2} \quad \text { for every } \quad x, y \in E .
$$

In [4, Theorem 2.4], it was shown that $\left(C W^{1,1}\right)$ is a necessary and sufficient condition on $(f, G)$ for the existence of a $C^{1,1}(X)$ convex extension $F$ of $f$ with $\nabla F=G$ on $E$.

Lemma 3.3 The 1-jet $(f, G)$ satisfies $\left(S C W^{1,1}\right)$ with constants $(c, M)$ on $E$ if and only if the 1-jet $(\tilde{f}, \tilde{G})=\left(f-\frac{c}{2}\|\cdot\|^{2}, G-c I\right)$ satisfies condition $\left(C W^{1,1}\right)$ with constant $M-c$ on $E$. 
Proof Assume first that $(f, G)$ satisfies $\left(S C W^{1,1}\right)$ with constants $(c, M)$ on $E$. We have

$$
\begin{aligned}
& \tilde{f}(x)-\tilde{f}(y)-\langle\tilde{G}(y), x-y\rangle-\frac{1}{2(M-c)}\|\tilde{G}(x)-\tilde{G}(y)\|^{2} \\
&= f(x)-f(y)-\langle G(y), x-y\rangle-\frac{c}{2}\|x-y\|^{2} \\
&-\frac{1}{2(M-c)}\left(\|G(x)-G(y)\|^{2}+c^{2}\|x-y\|^{2}+2 c\langle G(x)-G(y), y-x\rangle\right) \\
&= f(x)-f(y)-\langle G(y), x-y\rangle-\frac{c}{M-c}\langle G(x)-G(y), y-x\rangle \\
&-\frac{c M}{2(M-c)}\|x-y\|^{2}-\frac{1}{2(M-c)}\|G(x)-G(y)\|^{2} \geq 0 .
\end{aligned}
$$

Conversely, if $(\tilde{f}, \tilde{G})=\left(f-\frac{c}{2}\|\cdot\|^{2}, G-c I\right)$ satisfies condition $\left(C W^{1,1}\right)$ with constant $M-c$, we can write

$$
\begin{aligned}
f(x)- & f(y)-\langle G(y), x-y\rangle-\frac{c}{M-c}\langle G(x)-G(y), y-x\rangle \\
- & \frac{c M}{2(M-c)}\|x-y\|^{2}-\frac{1}{2(M-c)}\|G(x)-G(y)\|^{2} \\
= & \tilde{f}(x)-\tilde{f}(y)-\langle\tilde{G}(y), x-y\rangle+\frac{c}{2}\|x-y\|^{2} \\
& -\frac{c}{M-c}\langle\tilde{G}(x)-\tilde{G}(y), y-x\rangle+\frac{c^{2}}{M-c}\|x-y\|^{2} \\
& -\frac{c M}{2(M-c)}\|x-y\|^{2}-\frac{1}{2(M-c)} \\
& \times\left(\|\tilde{G}(x)-\tilde{G}(y)\|^{2}+c^{2}\|x-y\|^{2}+2 c\langle\tilde{G}(x)-\tilde{G}(y), x-y\rangle\right) \\
= & \tilde{f}(x)-\tilde{f}(y)-\langle\tilde{G}(y), x-y\rangle-\frac{1}{2(M-c)}\|\tilde{G}(x)-\tilde{G}(y)\|^{2} \geq 0 .
\end{aligned}
$$

Proposition 3.4 Let $F \in C^{1,1}(X)$ be such that $\operatorname{Lip}(\nabla F) \leq M$ and $g:=F-\frac{c}{2}\|\cdot\|^{2}$ is a convex function, where $c \in \mathbb{R}, M>0$ are two constants (if $c>0$, we call such a function $F$ a $C^{1,1}$ (globally) strongly convex function). Then the following properties hold.

(i) We have $M \geq c$ and if $M=c$, then $F$ is a quadratic function.

(ii) $g$ is of class $C^{1,1}(X)$ with $\operatorname{Lip}(\nabla g) \leq M-c$.

(iii) Assume that $M>c$. For every $x, y \in X$ we have

$$
\begin{aligned}
F(x) \geq F(y)+\langle\nabla F(y), x-y\rangle+\frac{c}{M-c}\langle\nabla F(x) & -\nabla F(y), y-x\rangle+\frac{c M}{2(M-c)}\|x-y\|^{2} \\
& +\frac{1}{2(M-c)}\|\nabla F(x)-\nabla F(y)\|^{2} .
\end{aligned}
$$

Therefore, according to Definition 3.1, $(F, \nabla F)$ satisfies condition $\left(S C W^{1,1}\right)$ on $X$ with constants $(c, M)$.

Proof (i) and (ii). Given $x, h \in X$, the fact that $\operatorname{Lip}(\nabla F) \leq M$ and the convexity of $g$ yield

$$
\begin{aligned}
0 & \leq g(x+h)+g(x-h)-2 g(x) \\
& =F(x+h)+F(x-h)-2 F(x)-\frac{c}{2}\left(\|x+h\|^{2}+\|x-h\|^{2}-2\|x\|^{2}\right) \\
& \leq(M-c)\|h\|^{2} .
\end{aligned}
$$

This shows that $c \leq M$ and, again by convexity of $g$, that $\operatorname{Lip}(\nabla g) \leq M-c$. Finally observe that if $M=c$, then $g$ is affine and therefore $F$ is a quadratic function. 
(iii). By virtue of (ii), the convexity of $g$ implies that $(g, \nabla g)$ satisfies condition $\left(C W^{1,1}\right)$ with constant $M-c$ on $X$. Thus, Lemma 3.3 gives the desired inequality.

Theorem 3.5 Let $E$ be an arbitrary subset of a Hilbert space $X$, let $f: E \rightarrow \mathbb{R}, G: E \rightarrow$ $X$ be two functions, and let $c \in \mathbb{R}, M>0$ be two constants. There exists a function $F \in C^{1,1}(X)$ such that $F=f, \nabla F=G$ on $E, \operatorname{Lip}(\nabla F) \leq M$, and $F-\frac{c}{2}\|\cdot\|^{2}$ is a convex function if and only if the jet $(f, G)$ satisfies condition $\left(S C W^{1,1}\right)$ with constants $(c, M)$ on $E$. In fact, $F$ can be defined by means of the formula

$$
\begin{aligned}
F & =\operatorname{conv}(g)+\frac{c}{2}\|\cdot\|^{2}, \\
g(x) & =\inf _{y \in E}\left\{f(y)+\langle G(y), x-y\rangle+\frac{M}{2}\|x-y\|^{2}\right\}-\frac{c}{2}\|x\|^{2}, \quad x \in X .
\end{aligned}
$$

Moreover, if $H$ is another function of class $C^{1,1}(X)$ satisfying the above properties, then $H \leq F$ on $X$.

Proof The necessity of the condition $\left(S C W^{1,1}\right)$ with constants $(c, M)$ on the jet $(f, G)$ follows immediately from Proposition 3.4 (iii). Conversely, assume that $(f, G)$ satisfies condition $\left(S C W^{1,1}\right)$ with constants $(c, M)$ on $E$. By Lemma 3.3, the jet $(\tilde{f}, \tilde{G})=\left(f-\frac{c}{2}\|\cdot\|^{2}, G-c I\right)$ satisfies condition $\left(C W^{1,1}\right)$ with constant $M-c$, and we can apply [4, Theorem 2.4] to obtain that $\tilde{F}=\operatorname{conv}(g)$, where

$$
\begin{aligned}
g(x) & =\inf _{y \in E}\left\{\tilde{f}(y)+\langle\tilde{G}(y), x-y\rangle+\frac{M-c}{2}\|x-y\|^{2}\right\} \\
& =\inf _{y \in E}\left\{f(y)+\langle G(y), x-y\rangle+\frac{M}{2}\|x-y\|^{2}\right\}-\frac{c}{2}\|x\|^{2}
\end{aligned}
$$

is convex and of class $C^{1,1}(X)$ with $\operatorname{Lip}(\nabla \tilde{F}) \leq M-c$ and $(\tilde{F}, \nabla \tilde{F})=(\tilde{f}, \tilde{G})$ on $E$. If we consider the function $F:=\tilde{F}+\frac{c}{2}\|\cdot\|^{2}$, Lemma 3.3 says that $(F, \nabla F)$ satisfies condition $\left(S C W^{1,1}\right)$ with constants $(c, M)$ on $X$ (because $(\tilde{F}, \nabla \tilde{F})$ satisfies $\left(C W^{1,1}\right)$ with constant $M-c$ on $X)$ and $(F, \nabla F)=(f, G)$ on $E$. It is obvious that $F-\frac{c}{2}\|\cdot\|^{2}$ is convex on $X$ and, by Remark 3.2(ii), $\operatorname{Lip}(\nabla F) \leq M$.

Finally, if $H$ is a function of class $C^{1,1}(X)$ such that $(H, \nabla H)=(f, G)$ on $E, \operatorname{Lip}(\nabla H) \leq M$ and $\tilde{H}:=H-\frac{c}{2}\|\cdot\|^{2}$ is convex, then it is easy to see (using the same calculations as in the proof of Proposition 3.4 (ii)) that $\operatorname{Lip}(\nabla \tilde{H}) \leq M-c$, and obviously $(\tilde{H}, \nabla \tilde{H})=(\tilde{f}, \tilde{G})$ on $E$. We thus have from [4, Theorem 2.4] that $\tilde{H} \leq \tilde{F}$ on $X$, and therefore $H \leq F$ on $X$.

\section{References}

[1] A. V. Akopyan and A. S. Tarasov, A constructive proof of Kirszbraun's theorem. Mat. Zametki 84(2008), no. 5, 781-784. https://doi.org/10.1134/S000143460811014X

[2] M. Aschenbrenner and A. Fischer, Definable versions of theorems by Kirszbraun and Helly. Proc. London Math. Soc (3) 102(2011), 468-502. https://doi.org/10.1112/plms/pdq029

[3] D. Azagra and C. Mudarra, Whitney Extension Theorems for convex functions of the classes $C^{1}$ and $C^{1, \omega}$. Proc. London Math. Soc. 114(2017), no. 1, 133-158. https://doi.org/10.1112/plms.12006

[4] D. Azagra, E. Le Gruyer, and C. Mudarra, Explicit formulas for $C^{1,1}$ and $C_{\mathrm{conv}}^{1, \omega}$ extensions of 1-jets in Hilbert and superreflexive spaces. J. Funct. Anal. 274(2018), 3003-3032.

https://doi.org/10.1016/j.jfa.2017.12.007 
[5] H. H. Bauschke, Fenchel duality, Fitzpatrick functions and the extension of firmly nonexpansive mappings. Proc. Amer. Math. Soc. 135(2007), 135-139.

https://doi.org/10.1090/S0002-9939-06-08770-3

[6] H. H. Bauschke and P.L. Combettes, Convex analysis and monotone operator theory in Hilbert spaces. 2nd ed., CMS Books in Mathematics, Springer, Cham, 2017.

https://doi.org/10.1007/978-3-319-48311-5

[7] H. H. Bauschke, S. M. Moffat, and X. Wang, Firmly nonexpansive mappings and maximally monotone operators: correspondence and duality. Set-Valued Anal. 20(2012), 131-153. https://doi.org/10.1007/s11228-011-0187-7

[8] H. H. Bauschke and X. Wang, The kernel average for two convex functions and its applications to the extension and representation of monotone operators. Trans. Amer. Math. Soc. 361(2009), 5947-5965. https://doi.org/10.1090/S0002-9947-09-04698-4

[9] H. H. Bauschke and X. Wang, Firmly nonexpansive and Kirszbraun-Valentine extensions: $a$ constructive approach via monotone operator theory. In: Nonlinear analysis and optimization I., Contemp. Math., 513, Israel Math. Conf. Proc., American Mathematical Society, Providence, RI, 2010, pp. 55-64. https://doi.org/10.1090/conm/513/10075

[10] J. Benoist and J.-B. Hiriart-Urruty, What is the subdifferential of the closed convex hull of a function?. SIAM J. Math. Anal. 27(1996), 1661-1679. https://doi.org/10.1137/S003614109465936

[11] Y. Benyamini and J. Lindenstrauss, Geometric nonlinear functional analysis. Amer. Math. Soc. Coll. Pubs. 48, American Mathematical Society, Providence, RI, 2000.

[12] Y. Brudnyi ad P. Shvartsman, Whitney's extension problem for multivariate $C^{1, \omega}$-functions. Trans. Amer. Math. Soc. 353(2001), 2487-2512. https://doi.org/10.1090/S0002-9947-01-02756-8

[13] J. M. Borwein and J. D. Vanderwerff, Convex functions: constructions, characterizations and counterexamples. Encyclopedia of Mathematics and its Applications, 109, Cambridge University Press, Cambridge, 2010. https://doi.org/10.1017/CB09781139087322

[14] A. Daniilidis, M. Haddou, E. Le Gruyer, and O. Ley, Explicit formulas for $C^{1,1}$ Glaeser-Whitney extensions of 1-fields in Hilbert spaces. Proc. Amer. Math. Soc. 146(2018), 4487-4495. https://doi.org/10.1090/proc/14012

[15] H. Federer, Geometric measure theory. Die Grundlehren der mathematischen Wissenschaften, Band, 153, Springer-Verlag, New York, 1969.

[16] C. Fefferman, A sharp form of Whitney's extension theorem. Ann. of Math. (2) 161(2005), no. 1, 509-577. https://doi.org/10.4007/annals.2005.161.509

[17] C. Fefferman, Whitney's extension problem for $C^{m}$. Ann. of Math. (2) 164(2006), no. 1, 313-359. https://doi.org/10.4007/annals.2006.164.313

[18] C. Fefferman, Whitney's extension problems and interpolation of data. Bull. Amer. Math. Soc. (N.S.) 46(2009), no. 2, 207-220. https://doi.org/10.1090/S0273-0979-08-01240-8

[19] M. Ghomi, Strictly convex submanifolds and hypersurfaces of positive curvature. J. Differential Geom. 57(2001), 239-271.

[20] M. Ghomi, The problem of optimal smoothing for convex functions. Proc. Amer. Math. Soc. 130(2002), no. 8, 2255-2259. https://doi.org/10.1090/S0002-9939-02-06743-6

[21] F. Grünbaum and E.H. Zarantonello, On the extension of uniformly continuous mappings. Michigan Math. J. 15(1968), 65-74.

[22] B. Kirchheim, J. Kristensen, Differentiability of convex envelopes. C. R. Acad. Sci. Paris Sér. I Math. 333(2001), no. 8, 725-728. https://doi.org/10.1016/S0764-4442(01)02117-6

[23] M. D. Kirszbraun, Über die zusammenziehenden und Lipschitzschen Transformationen. Fund. Math. 22(1934), 77-108.

[24] E. Le Gruyer, Minimal Lipschitz extensions to differentiable functions defined on a Hilbert space. Geom. Funct. Anal 19(2009), 1101-1118. https://doi.org/10.1007/s00039-009-0027-1

[25] E. Le Gruyer, Thanh-Viet Phan, Sup-Inf explicit formulas for minimal Lipschitz extensions for 1-fields on $\mathbb{R}^{n}$. J. Math. Anal. Appl. 424(2015), 1161-1185. https://doi.org/10.1016.j.jmaa.2014

[26] E. J. Mickle, On the extension of a transformation. Bull. Amer. Math. Soc. 55(1949), 160-164. https://doi.org/10.1090/S0002-9904-1949-09189-9

[27] S. Reich and S. Simons, Fenchel duality, Fitzpatrick functions and the Kirszbraun-Valentine extension theorem. Proc. Amer. Math. Soc. 133(2005), 2657-2660. https://doi.org/10.1090/S0002-9939-05-07983-9

[28] T. Rockafellar, Convex analysis. Princeton Mathematical Series, 28, Princeton University Press, Princeton, NJ, 1970.

[29] T. Rockafellar and R.J.-B. Wets, Variational analysis. Springer, New York, 1998.

[30] F. A. Valentine, A Lipschitz condition preserving extension for a vector function. Amer. J. Math. 67(1945), 83-93. https://doi.org/10.2307/2371917 
[31] M. Yan, Extension of convex function. J. Convex Anal. 21(2014) no. 4, 965-987.

[32] J. C. Wells, Differentiable functions on Banach spaces with Lipschitz derivatives. J. Differential Geometry 8(1973), 135-152.

ICMAT (CSIC-UAM-UC3-UCM), Departamento de Análisis Matemático y Matemática Aplicada, Facultad Ciencias Matemáticas, Universidad Complutense, 28040, Madrid, Spain

e-mail: azagra@mat.ucm.es

INSA de Rennes \& IRMAR, 20, Avenue des Buttes de Coësmes, CS 70839 F-35708, Rennes Cedex 7, France e-mail: Erwan.Le-Gruyer@insa-rennes.fr

ICMAT (CSIC-UAM-UC3-UCM), Calle Nicolás Cabrera 13-15. 28049 Madrid, Spain

e-mail: carlos.mudarra@icmat.es 